\title{
Adsorbate-induced substrate relaxation and the adsorbate-adsorbate interaction
}

\author{
R. Brako and D. Šokčević \\ Rudjer Bošković Institute, P.O. Box 1016, 10001 Zagreb, Croatia
}

(February 26, 2018)

\begin{abstract}
We formulate the theory of the perturbation caused by an adsorbate upon the substrate lattice in terms of a local modification of the interatomic potential energy around the adsorption site, which leads to the relaxation of substrate atoms. We apply the approach to CO chemisorption on closepacked metal surfaces, and show that the adsorbate-adsorbate interaction and a variety of other properties can be well described by a simple model.
\end{abstract}

PACS numbers: 68.35.-p, 68.35.Md, 82.65.My

Several direct and indirect (through-the-substrate) mechanisms can lead to an effective interaction between adsorbates on metal surfaces. The possible role of adsorbate-induced substrate relaxation was considered some time ago by Lau and Kohn [1,2], using an elastic continuum model of the surface. They concluded that the resulting interaction was repulsive between identical adsorbates, varied as $\rho^{-3}$ with separation $\rho$, and was inversely proportional to the shear modulus of the substrate. For adsorbates separated by a few atomic spacings, however, the continuum theory may not be valid. More recently, in a series of papers Kevan et al. [3 7] have determined adsorbate-adsorbate interaction energies for $\mathrm{CO}$ at several metal surfaces, using a transfer-matrix analysis of thermal desorption spectra. They have also qualitatively discussed the adsorbate-induced strain as a possible mechanism of the adsorbate-adsorbate interaction at intermediate range, i.e., several substrate atoms apart.

The potential energy of the atomic lattice of a solid in the harmonic approximation can be written as

$$
V=\sum_{i, j, \mu, \nu} x_{\mu}(i) D_{\mu \nu}(i, j) x_{\nu}(j)
$$

where $x_{\mu}(i)$ is the $\mu$-th component of the displacement of the $i$-th atom from the equilibrium position. Now assume that an impurity is created by replacing one atom with a different species. (In the general discussion we talk about an "impurity", although we are primarily interested in the chemisorption case, where the adsorbate also introduces additional degrees of freedom. The generalization to the latter case is straightforward.) The potential energy after the replacement can again be expressed in the form (1i), but the new equlibrium positions $x_{\mu}^{\prime}(i)$ are in general different:

$$
x_{\mu}^{\prime}(i)=x_{\mu}(i)+\Delta x_{\mu}(i)
$$

The new dynamical matrix $D_{\mu \nu}^{\prime}(i, j)$ is also different, and a constant term appears which shifts the energy minimum. Using (2), the potential energy of the system after the impurity has been introduced can be expressed in the coordinates $x_{\mu}(i)$ :

$$
V^{\prime}=\sum_{i, j, \mu, \nu} x_{\mu}(i) D_{\mu \nu}^{\prime}(i, j) x_{\nu}(j)+\sum_{i, \mu} F_{\mu}(i) x_{\mu}(i)+V_{0}^{\prime},
$$

i.e., the dynamical matrix is modified, and linear (force) and constant (energy shift) terms appear.

The foregoing considerations depend only upon the assumed stability of the solid, i.e., the existence of the minimum of the potential energy. For our application to chemisorption, we specifically assume the following properties: (a) the force terms $F_{\mu}(i)$ are nonzero only for a small number of substrate atoms around the adsorption site; (b) similarly, only a few elements of the dynamical matrix $D_{\mu \nu}(i, j)$ change, if any; (c) the effect is linear, so that the chemisorption of another molecule (and, consequently, of a third, a fourth, etc.) can be described by the same set of parameters, of course centered around the new adsorption site. The condition (c) is rather restrictive and excludes systems which reconstruct at large adsorbate coverage, as well as those where other interactions are important, such as the direct adsorbateadsorbate repulsion, the electrostatic dipole-dipole interaction, or the "chemical" competition for the same electronic orbitals in the substrate. Estimates, however, show that such interactions are usually weak and shortranged, while we are interested in medium-range interactions (second nearest neighbor and beyond) of nonionic adsorbates.

In this paper we take into account the in-plane relaxation within the first atomic layer of the substrate induced by a chemisorbed species. The relaxation in the perpendicular direction can, of course, be equally strong, but it does not contribute much to the effective adsorbate-adsorbate interaction and to the adsorbateinduced surface stress. Also, we do not consider the coupling to internal adsorbate coordinates, which was discussed in Ref. [8] in an application to the damping of adsorbate vibrations.

For a hexagonal layer of atoms, we write the potential energy as

$$
V=\frac{1}{2} \sum_{i} \sum_{j=1}^{6} \frac{1}{2} K_{1}\left[\hat{\mathbf{r}}_{i j} \cdot\left(\mathbf{r}_{i}-\mathbf{r}_{j}\right)\right]^{2}+\frac{1}{2} \sum_{i} K_{2} \mathbf{r}_{i}^{2},
$$


where $\mathbf{r}_{\mathbf{i}}=\left(\mathbf{x}_{\mathbf{i}}, \mathbf{y}_{\mathbf{i}}\right)$ is the in-plane displacement from the equlibrium position of the $i$-th atom. The term $K_{1}$ describes a central atom-atom interaction, and the term $K_{2}$ binds atoms to their equilibrium positions, simulating the interaction to lower atomic layers. Without it, the model would be too "soft" to long-wavelength perturbations. The trade-off is that the lowest phonon frequency becomes finite, i.e., there are no true "acoustic" modes, but this has little influence on the relaxation energy and other static quantities calculated in this work.

Now assume that an atom or a molecule chemisorbs on top of the atom $i=0$, and that the induced change of the potential energy is

$$
\begin{aligned}
V^{\prime}= & \sum_{j=1}^{6} \frac{1}{2} \Delta K_{1}\left[\hat{\mathbf{r}}_{0 j} \cdot\left(\mathbf{r}_{0}-\mathbf{r}_{j}\right)\right]^{2} \\
& -\sum_{j=1}^{6} \frac{k_{2}}{\alpha} \hat{\mathbf{r}}_{0 j} \cdot\left(\mathbf{r}_{0}-\mathbf{r}_{j}\right)+V_{0}^{\prime},
\end{aligned}
$$

where the first term describes the change of the force constant between the atom 0 and the six surrounding atoms, and the second is a linear force term. In the following we drop the $\Delta K_{1}$ and $V_{0}^{\prime}$ terms, which are beyond the level of accuracy assumed in this work, although, in general, the $\Delta K_{1}$ term ought to be included.

The definition of the chemisorption-induced surface stress $\tau$ is [9]

$$
\delta W=A \tau_{x} \delta \epsilon_{x},
$$

where $A$ is the surface area, $\delta \epsilon_{x}$ the strain, and $\delta W$ the difference of the work involved in straining a clean surface and a surface with adsorbates. In our model, we obtain

$$
\tau_{x}=-\frac{k_{2}}{a \alpha} 2 \sqrt{3} \theta,
$$

where $\theta$ is the adsorbate coverage. (If $\Delta K_{1}$ is different from zero, an additional factor of order unity appears in the above expression.)

Irrespective of the sign of the force term $k_{2} / \alpha$, there is always an energy gain due to the relaxation of the surrounding atoms, i.e., the minimum of $V+V^{\prime}$ is less than zero. If another molecule adsorbs on a nearby site, the forces induced by the two adsorbates act in opposite directions and the relaxation is less complete than with adsorbates far apart, which leads to an effective interaction. We calculate the interaction energies by comparing the minimum of the potential energy $V+V^{\prime}$ for a single adsorbed molecule with the minimum for two molecules adsorbed on, in turn, second, third, fourth, and fifth nearest-neighbor sites, see Fig. 1 1(a). (Throughout the paper we assume that there is a strong repulsion between first nearest neighbor adsorbates caused by "chemical" effects, and do not consider them.)
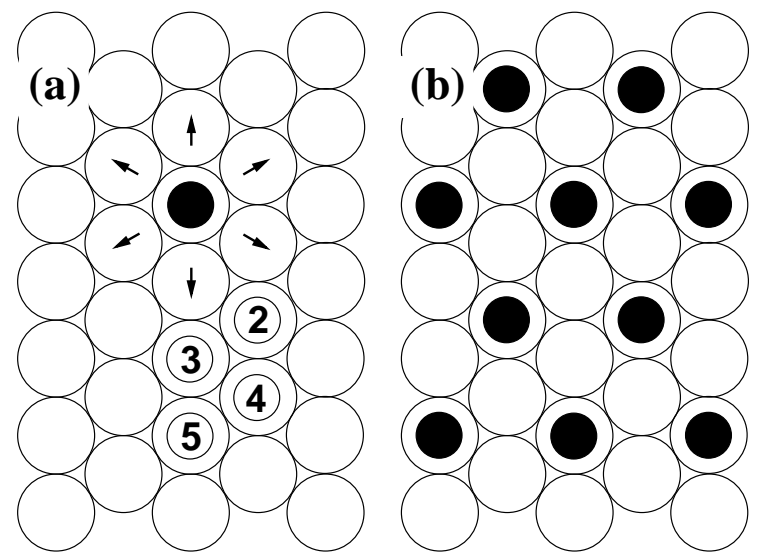

FIG. 1. (a) Chemisorption of CO, black circle, into an on-top site on $\operatorname{Pt}(111)$. The chemisorbate causes a relaxation of the adjacent $\mathrm{Pt}$ atoms, denoted by arrows, which propagates to further first-layer atoms via elastic forces. The neighboring adsorption sites are indicated by numbers. (b) The regular $(\sqrt{3} \times \sqrt{3}) \mathrm{R} 30^{\circ}$ structure at a coverage $\theta=0.33$. Owing to symmetry, no relaxation of substrate atoms along the surface plane is possible.

We first consider the chemisorption of $\mathrm{CO}$ on a $\mathrm{Pt}(111)$ surface. There is a large amount of tensile stress in the first atomic layer of the clean $\mathrm{Pt}(111)$ surface [10,11]. Although the surface does not reconstruct at room temperature, a reconstruction is observed at high temperatures in the presence of saturated $\mathrm{Pt}$ vapor 10]. CO adsorbs initially into on-top sites of $\operatorname{Pt}(111)$, but the energy difference for the adsorption into bridge sites is obviously small, since some bridge adsorbates are found already at coverages $\theta$ above 0.15 [12]. Some authors report (Ref. [13] and references therein) that a regular $(\sqrt{3} \times \sqrt{3})$ R30 $0^{\circ}$ structure at a coverage $\theta=1 / 3$ is formed, Fig. If(b), but others claim that the densest structure of exclusively on-top adsorbates exists at $\theta=0.29$ [14], and that further chemisorption occurs into bridge sites. The regular structure at $\theta=0.5$ contains an equal number of on-top and bridge adsorbates.

We have chosen the values of the parameters $K_{1}, K_{2}$, and $k_{2} / \alpha$ which give good agreement with experimental data on adsorbate-induced surface stress [9] and with low-coverage interaction energies [4], as shown in Table [. We had to choose a rather small value for the force constant $K_{1}$ between atoms in the first surface layer. (The value of $K_{2}$ has little effect on the results. The much larger value of $K_{1}$ used in a similar model in Ref. [8] was an overestimate.) The reduction from bulk values is characteristic of many close-packed noble-metal surfaces [15], but the reduction we find is larger than suggested in the surface phonon calculation in Ref. [16]. Consequently, the highest resulting vibrational frequency of two-dimensional phonons of the first surface layer is about $5 \mathrm{meV}$, by about a factor of two smaller than the frequency of surface phonons along the edge of the Bril- 
louin zone of around $10 \mathrm{meV}$ calculated in Ref. 16]. As discussed above, it is possible that part of the softening is localized around the adsorption site only, the term $\Delta K_{1}$ in Eq. (5). The values in Table If show that the repul-

TABLE I. Interaction energies $W_{n \mathrm{NN}}$ (in K) and the induced surface stress $\tau$ (in $\mathrm{N} / \mathrm{m}$ ) for on-top adsorption of $\mathrm{CO}$ on $\mathrm{Pt}(111)$. $W_{n \mathrm{NN}}=2 E_{0}-E_{n \mathrm{NN}}$, where $E_{0}$ is the relaxation energy of a single $\mathrm{CO}$ molecule, $E_{n \mathrm{NN}}$ is the relaxation energy for two $\mathrm{CO}$ molecules adsorbed at the $n$-th nearest-neighbor sites, etc. Theoretical values are calculated using $K_{1}=4 \mathrm{~N} / \mathrm{m}, K_{2}=2.5 \mathrm{~N} / \mathrm{m}, k_{2} / \alpha=0.3 \times 10^{-9} \mathrm{~N}$. The nearest-neighbor distance is $a=2.76 \AA$.

\begin{tabular}{lclllll}
\hline \hline & $W_{2 \mathrm{NN}}$ & $W_{3 \mathrm{NN}}$ & $W_{4 \mathrm{NN}}$ & $W_{5 \mathrm{NN}}$ & $E_{0} / 6$ & $\tau$ \\
\hline Theory & 194 & 314 & 108 & 119 & 231 & 1.24 \\
Experiment & $120^{\mathrm{a}}$ & $400^{\mathrm{a}}$ & $236^{\mathrm{a}}$ & & $452^{\mathrm{b}}$ & $1.2^{\mathrm{c}}$ \\
\hline \hline
\end{tabular}

${ }^{\mathrm{a}}$ Ref. 4. low CO coverage.

${ }^{\mathrm{b}}$ Ref. 3 , 3 , CO coverage of 0.33

${ }^{\mathrm{c}}$ Ref. [9], CO coverage of 0.33 .

sive interaction is strong between $\mathrm{CO}$ adsorbed on sites lying along rows of substrate atoms, and weaker for adsorbates separated by hollows, even if they are less far apart. In our opinion, the rather large interaction energy between fourth nearest neighbor adsorbates in Ref. 沺] is influenced by the contributions from more distant sites, which were not included in their analysis.

Clean nickel (111) surfaces do not reconstruct. Unlike some earlier claims, it is now accepted that at low temperature $\mathrm{CO}$ chemisorbs initially into threefold hollow sites [17, 18]. At room temperature, some bridge and on-top sites seem to be occupied even at low coverages [18]. At a coverage $\theta=0.33, \mathrm{CO}$ forms a regular $(\sqrt{3} \times \sqrt{3})$ R30 $0^{\circ}$ structure 19], but it is not clear whether the molecules adsorb into fcc or hcp positions. At $\theta=0.5$, a regular $\mathrm{c}(4 \times 2)-2 \mathrm{CO}$ structure is formed, in which an equal number of fcc and hcp sites is occupied [19 21], Fig. 2(b). The top layer of Ni atoms shows buckling in that nonequivalent atoms have different vertical relaxation. The $\mathrm{CO}$ molecules are slightly tilted from the direction perpendicular to the surface. The initial heat of adsorption at room temparature is $130 \mathrm{~kJ} / \mathrm{mol}$ [22]. It decreases slowly at first, to around $122 \mathrm{~kJ} / \mathrm{mol}$ at $\theta=0.33$ and $112 \mathrm{~kJ} / \mathrm{mol}$ at $\theta=0.5$. However, the fact that not all adsorption sites are equivalent, and the rather large standard deviation of experimental data make the interpretation of the coverage dependence of the heat of adsorption uncertain.

We describe the first layer of $\mathrm{Ni}$ atoms by the same potential as for $\mathrm{Pt}$, Eq. (4). The interaction terms are similar to Eq. (5), but the adsorbate is in a threefold hollow site and the sums run over the three surrounding $\mathrm{Ni}$ atoms (Fig. 2). The induced surface stress is

$$
\tau=-\frac{k_{2}}{\alpha a} \theta,
$$
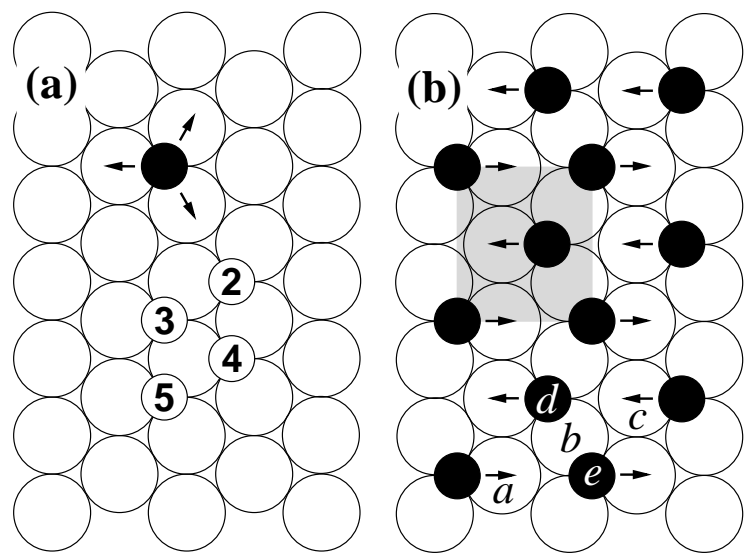

FIG. 2. (a) Chemisorption of CO, black circle, into a three-fold hollow site on $\mathrm{Ni}(111)$. The relaxation of the adjacent $\mathrm{Ni}$ atoms is denoted by arrows. The numbers indicate possible adsorption sites of the same kind, either fcc or hcp. (b) The regular $\mathrm{c}(4 \times 4)-2 \mathrm{CO}$ structure on $\mathrm{Ni}(111)$ at a coverage $\theta=0.5$. An elementary cell (grey area) contains one adsorbate in the fcc and one in the hcp position, $d$ and $e$. The relaxation of $\mathrm{Ni}$ atoms in intermediate rows, $a$ and $c$, is denoted by arrows.

where the nearest-neighbor $\mathrm{Ni}-\mathrm{Ni}$ distance is $a=2.49 \AA$. From the experimental data $\tau=-0.55 \mathrm{~N} / \mathrm{m}$ at $\theta=$ 0.33 [23] we have estimated $k_{2} / \alpha=1 \times 10^{-10} \mathrm{~N}$. In a LEED analysis of the $\mathrm{c}(4 \times 2)-2 \mathrm{CO}$ structure which forms at $\theta=0.5$, Mapledoram et al. 20] found that the lateral displacement of the $\mathrm{Ni}$ atoms next to an adsorbate, $a$ and $c$ in Fig. 2(b), was $0.03 \AA$. We have reproduced this value by taking $K_{1}=6 \mathrm{~N} / \mathrm{m}$ and $K_{2}=2 \mathrm{~N} / \mathrm{m}$. (As before, the value of $K_{2}$ is of lesser importance.) This is a considerable reduction from the bulk values, but not as large as for the $\mathrm{Pt}(111)$ surface, in agreement with the fact that the $\mathrm{Ni}(111)$ surface seems less prone to reconstruct than $\mathrm{Pt}(111)$. The energy gain per adsorbate is $22 \mathrm{~K}$.

Using these values of the parameters, the relaxation energy for a single adsorbate is only $42 \mathrm{~K}$, which is 30 times smaller than that we have found for $\mathrm{CO} / \mathrm{Pt}(111)$. (This reduction can be well estimated treating each surrounding $\mathrm{Ni}$ atom as an independent oscillator: The force $k_{2} / \alpha$ is three times smaller than in the $\mathrm{CO} / \mathrm{Pt}(111)$ case, the $\mathrm{Ni}-\mathrm{Ni}$ force constant is $50 \%$ larger, and there are 3 surrounding atoms instead of 6.) Furthermore, the displacements of $\mathrm{Ni}$ atoms around the hollow adsorption site are not along chains of atoms, and do not cause a large displacement of other atoms. The calculated effective interaction energies for second nearest neighbors and beyond are therefore only a few $\mathrm{K}$, too small to be observable. In our opinion, the interaction energy of $100 \mathrm{~K}$ between second neighbor adsorbates suggested by Skelton et al. [5] is either a combined result of other mechanisms or an artefact of their procedure. We note that an earlier study [24] reported that there was essentially no interaction already between second neighbor adsorbates. 
Interaction energies have also been determined for $\mathrm{CO}$ chemisorbed on $\mathrm{Rh}$ [7,25] and $\mathrm{Cu}$ [6] surfaces. We discuss these systems only qualitatively, since the proposed values are still uncertain, and there is no quantitative data on other adsorbate-induced properties. Wei et al. [7] estimated that $W_{2}=-100 \mathrm{~K}$ and $W_{3}=150 \mathrm{~K}$ for the on-top chemisorbed $\mathrm{CO}$ on $\mathrm{Rh}(111)$. An earlier measurement by Payne et al. 25 reported $W_{2}=170 \mathrm{~K}$ and $W_{3}=-85 \mathrm{~K}$. In our model, the relative magnitudes of interaction energies for on-top adsorbates on fcc (111) surfaces are always similar to those found for $\mathrm{CO} / \mathrm{Pt}(111)$. In particular, we expect large repulsion between third nearest neighbor adsorbates which lie along a chain of substrate atoms. In this respect the values proposed in Ref. [7] seem more probable, although the origin of the attractive $W_{2}$ (if it is real) is not clear. For the on-top $\mathrm{CO}$ on $\mathrm{Cu}(111)$, the same authors found $W_{2}=107 \mathrm{~K}$, $W_{3}>800 \mathrm{~K}, W_{4}=155 \mathrm{~K}[6]$. The value of $W_{3}$ seems too large compared with the other two, but otherwise the results are quite similar to $\mathrm{CO} / \mathrm{Pt}(111)$.

The square lattice of the first layer of the (100) surface of the fcc bulk is not well described by a purely pairwise potential similar to Eq. (4), since in the absence of angular force constants and of coupling to lower layers, only the ad hoc term $K_{2}$ ensures the stability. Nevertheless, the trends in the interaction energies between on-top adsorbates can be deduced by analogy with (111) surfaces. We expect a repulsive interaction between third nearest neighbor adsorbates which lie on the same chain of atoms, and no interaction between second nearest neighbors which lie diagonally on different chains, because the forces on adjacent substrate atoms are orthogonal. In the latter case, even a weak attraction is possible owing to partly collinear displacements induced by the two adsorbates on more distant surface atoms. Indeed, the values $W_{2}=0$ and $W_{3}=400 \mathrm{~K}$ were found for $\operatorname{Rh}(100)$ [7], while the values suggested for $\mathrm{Cu}(100)$ were $W_{2}=-33 \mathrm{~K}$ and $W_{3}=13 \mathrm{~K}[6]$. It is interesting that the continuum elastic theory [2] also gives a strong repulsion in the $\langle 110\rangle$ direction and possibly a weak attraction in the $\langle 100\rangle$ direction between adsorbates on (100) surfaces of noble metals.

We have shown that the adsorbate-induced substrate relaxation leads to an adsorbate-adsorbate interaction which is quite long-ranged and has a nonmonotonic distance dependence, being particularly large between molecules adsorbed in on-top positions along a chain of surface atoms. The same mechanism also leads to other observable effects, such as the adsorbate-induced surface stress and the relaxation displacement of substrate atoms. Quantitative agreement with experiment can be obtained for CO adsorbed on $\mathrm{Pt}(111)$ and some other surfaces using a simple model, assuming that force constants between atoms in the first surface layer are considerably weaker than in the bulk, which is a known property of many close-packed noble metal surfaces. The results em- phasize the importance of allowing the full substrate relaxation in the first-principle calculations of chemisorption on metal surfaces.

This work was supported by the Ministry of Science and Technology of the Republic of Croatia under contract No. 00980101.

[1] K. H. Lau and W. Kohn, Surface. Sci. 65, 607 (1977).

[2] K. H. Lau, Solid State Commun. 28, 757 (1978).

[3] S. D. Kevan, J. Molecular Catalysis A: Chemical 131, 19 (1998).

[4] D. C. Skelton, D. H. Wei, and S. D. Kevan, Surf. Sci. 320, 77 (1994).

[5] D. C. Skelton, D.-H. Wei, and S. D. Kevan, Surf. Sci. 37064 (1997).

[6] D. H. Wei, D. C. Skelton, and S. D. Kevan, Surf. Sci. 326, 167 (1995).

[7] D. H. Wei, D. C. Skelton, and S. D. Kevan, Surf. Sci. 381, 49 (1997).

[8] R. Brako and D. Šokčević, Surf. Sci. 401, L388 (1998).

[9] H. Ibach, Surf. Sci. Rep. 29, 193 (1997).

[10] M. Hohage, T. Michely, and G. Comsa, Surf. Sci. 337, 249 (1995).

[11] G. Boisvert, L. J. Lewis, and M. Scheffler, Phys. Rev. B 57, 1881 (1998).

[12] J. Yoshinobu and M. Kawai, Surf. Sci. 363, 105 (1996).

[13] Y. Y. Yeo, L. Vattuone, and D. A. King, J. Chem. Phys. 106, 392 (1997).

[14] M. Tüshaus, E. Schweizer, P. Hollins, and A. M. Bradshaw, J. Electron. Spectrosc. Related Phenomena 44, 305 (1987).

[15] V. Bortolani, F. Ercolessi, E. Tosatti, A. Franchini, and G. Santoro, Europhys. Lett. 12, 149 (1990).

[16] V. Bortolani, A. Franchimi, G. Santoro, J. P. Toennies, Ch. Wöll, and G. Zhang, Phys. Rev. B 40, 3524 (1989).

[17] J. G. Chen, W. Erley, and H. Ibach, Surf. Sci. Lett. 223, L891 (1989).

[18] G. Held, J. Schuler, W. Sklarek, and H.-P. Steinrück, Surf. Sci. 398, 154 (1998).

[19] L. Becker, S. Aminpirooz, B. Hillert, M. Pedio, J. Haase, and D. L. Adams, Phys. Rev. B 47, 9710 (1993).

[20] L. D. Mapledoram, M. P. Bessent, A. Wander, and D. A. King, Chem. Phys. Lett. 228, 527 (1994).

[21] P. T. Sprunger, F. Besenbacher, and I. Stensgaard, Chem. Phys. Lett. 243, 439 (1995).

[22] J. T. Stuckless, N. Al-Sarraf, C. Wartnaby, and D. A. King, J. Chem. Phys. 99, 2202 (1993).

[23] A. Grossmann, W. Erley, and H. Ibach, Surf. Sci. 313, 209 (1994).

[24] O. L. J. Gijzeman, M. M. J. Zandvoort, F. Labohm, J. A. Vleigenthart, and G. Jongert, J. Chem. Soc. Faraday Trans. II 80, 771 (1984).

[25] S. H. Payne, H. J. Kreuzer, K. A. Peterlinz, T. J. Curtiss, C. Uebing, and S. J. Sibener, Surf. Sci. 272, 102 (1992). 